\title{
COMPARISON OF IOT DEVICES ARCHITECTURE IN HOME NETWORK
}

\author{
Takashi Murakami ${ }^{1}$, Keiichi Teramoto ${ }^{2}$, Ritsuko Kanazawa ${ }^{3}$, Ryo Hasegawa $^{4}$, Tetsushi Matsuda ${ }^{5}$, \\ Kanji Mizuno ${ }^{5}$, Dai Ando ${ }^{6}$, Yasuo Sugawara ${ }^{7}$, Yoko Masuda ${ }^{7}$, Yudai Kawasaki ${ }^{7}$, Yoichi Masuda ${ }^{1}$, \\ Moe Hamamoto $^{1}$, Hiroshi Sugimura ${ }^{8}$ and Masao Isshiki ${ }^{8}$ \\ ${ }^{l}$ ECHONET Consortium / Panasonic Corporation, Shimbashi TS Bldg. 4F, 1-22-5 Nishishinbashi, Minato-ku, \\ Tokyo 105-0003 Japan \\ ${ }^{2}$ ECHONET Consortium, Shimbashi TS Bldg. 4F, 1-22-5 Nishishinbashi, Minato-ku, Tokyo 105-0003 Japan \\ ${ }^{3}$ ECHONET Consortium / Hitachi, Ltd., Shimbashi TS Bldg. 4F, 1-22-5 Nishishinbashi, Minato-ku, \\ Tokyo 105-0003 Japan \\ ${ }^{4}$ ECHONET Consortium / Hitachi Appliance, Inc., Shimbashi TS Bldg. 4F, 1-22-5 Nishishinbashi, Minato-ku, \\ Tokyo 105-0003 Japan \\ ${ }^{5}$ ECHONET Consortium / Mitsubishi Electric Corporation, Shimbashi TS Bldg. 4F, 1-22-5 Nishishinbashi, \\ Minato-ku, Tokyo 105-0003 Japan \\ ${ }^{6}$ ECHONET Consortium / Nippon Telegraph and Telephone Corporation, Shimbashi TS Bldg. 4F, 1-22-5 Nishishinbashi, \\ Minato-ku, Tokyo 105-0003 Japan \\ ${ }^{7}$ ECHONET Consortium / Sharp Corporation, Shimbashi TS Bldg. 4F, 1-22-5 Nishishinbashi, Minato-ku, \\ Tokyo 105-0003 Japan \\ ${ }^{8}$ Kanagawa Institute of Technology, 1030 Shimoogino, Atsugi-city, Kanagawa 243-0292 Japan
}

\begin{abstract}
Recently, the number of IoT devices has increased, and this trend has been extended to home appliances (e.g. air conditioners, washing machines, refrigerators, etc.) and equipment (e.g. storage batteries, photovoltaics, etc.). There are mainly two architectures regarding to IoT conversion of the appliances installed in these houses. Specifically, they are "IoT devices connect to a server on the Internet via a controller" and "IoT devices connect to a server on the Internet directly". In this paper, these architectures are compared and it is shown that what kind of usage each architecture is effective.
\end{abstract}

\section{KEYWORDS}

IoT, Architecture, Home Network, ECHONET Lite, Cloud, Service

\section{INTRODUCTION}

In recent years, as Internet technology and network technology have progressed, standard transmission media (Ethernet, Wi-Fi, Bluetooth etc.) that can be used for home networks has been spreading. Therefore, even in the home, home appliances and equipment (hereinafter referred to devices) that had been operating as standalone in the past have network functions installed and have become IoT devices. And home network (Biljana L. and Kire V., 2017), (Charlie, W. et al, 2014) can be built with IoT devices. Now that various services such as the home energy management system (HEMS) and remote control from outside the home etc. are being introduced.

It is important how to convert devices in home to IoT. Now there are roughly two possible architectures to convert each device in home into IoT. The first is that the device itself is not connected directly to the service on the Internet but is connected to the service on the Internet via the controller installed in the home to construct the system (Figure 1 (a)). At this time, standard communication specifications (e.g. ECHONET Lite (ISO/IEC 14543-4-3, IEC 62394) (ECHONET, 2019), KNX (ISO/IEC 14543-4-3) (KNX, 2019), ZigBee (ZigBee, 2019) etc.) or proprietary specifications are used as communication specifications between the controller and the devices. In this paper, home network is focused on and since it is common that a lot of people often use device of multiple vendors at each home, communication specifications between controllers and devices are 
considered to use standard communication specifications. The controller has a function of controlling each device and a function of monitoring each device, and connects to a server on the Internet. A server on the Internet can cooperate with various business operators and provide services to users. The second is that the device itself connects directly to the service on the Internet (Figure 1 (b)). In many cases, the device can be connected only to a server operated by a vendor that manufactures the device. As a connection method, there are a method of connecting to a server via a router installed in the house, a method of connecting via a communication network such as LPWA and LTE, and so on. Each vendor's server cooperates with service providers to provide services to users.

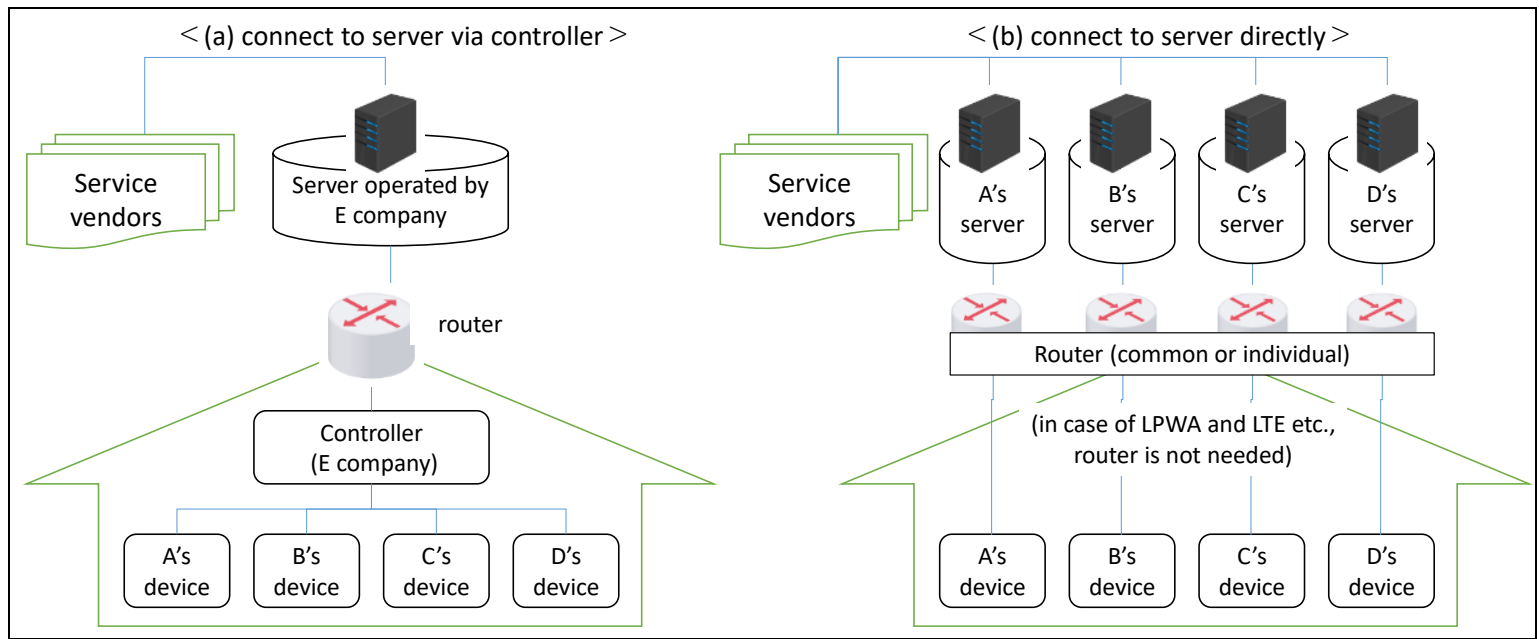

Figure 1. Architectures of home network

Depending on the services offered to users and the objectives of converting the devices to IoT, the architecture to be selected is different. In this paper, both architectures are compared from the viewpoint of realizing various services using home network system. There are various cooperation methods between servers on the Internet, such as WebAPI, MQTT and so on, but in this paper, in order to clarify the architecture of IoT devices as a main focus, the methods between servers are simply abstracted.

\section{ARCHITECTURES OF HOME NETWORK}

Particularly in Japan, home networks are spreading with HEMS as a trigger. From the perspective of further spreading in the future, the viewpoints of "system construction", "realization of various kinds of services", and "introduction / operation cost" are important. The evaluation of both architectures is summarized from these viewpoints.

\subsection{System Construction}

Items to compare architectures in system construction are shown in Figure 2, and outline of the comparison is shown in Table 1. As items to be compared, "Connection between service providers and server", "Introduction of controller", "Introduction of IoT device" can be mentioned. 


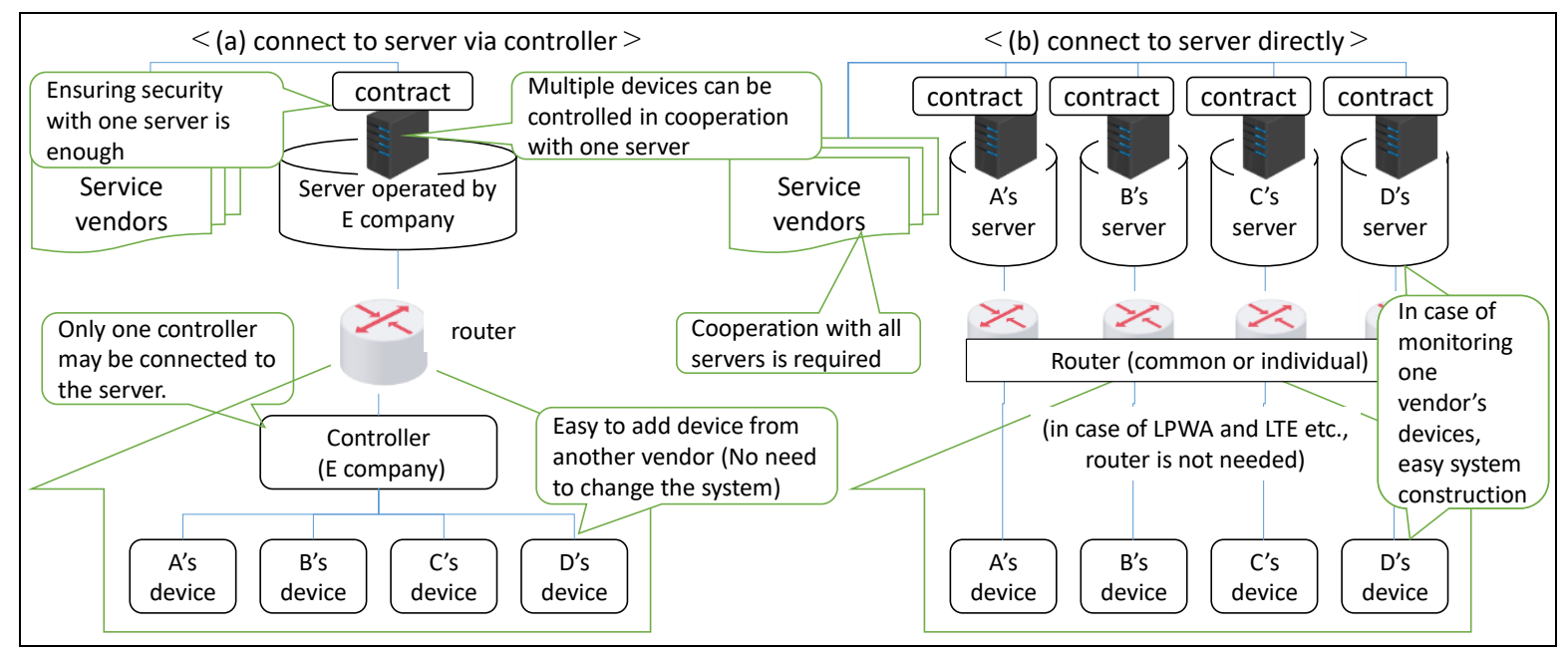

Figure 2. Comparison of architecture related to "system construction"

Table 1. Outline of comparison of architecture related to "system construction"

\begin{tabular}{|c|c|c|}
\hline & (a) Connect to server via controller & (b) Connect to server directly \\
\hline $\begin{array}{l}\text { Connection between servi } \\
\text { providers and server }\end{array}$ & $\begin{array}{l}\text { For each house, the server and the } \\
\text { service providers can establish a system } \\
\text { with } 1: \mathrm{N} \text { connection }\end{array}$ & $\begin{array}{l}\text { For each house, the servers and the } \\
\text { service providers can establish a } \\
\text { system with } \mathrm{M} \text { : } \mathrm{N} \text { connection }\end{array}$ \\
\hline Introduction of controller & $\begin{array}{l}\text { Since the controller terminates the } \\
\text { devices in the home, there is no need to } \\
\text { replace the system such as the controller } \\
\text { and the server in case of adding or } \\
\text { changing the device. }\end{array}$ & $\begin{array}{l}\text { No controller is needed. } \\
\text { When device vendors monitor or } \\
\text { control only their own devices, it is } \\
\text { possible to construct a system } \\
\text { without a controller. }\end{array}$ \\
\hline Introduction of IoT device & $\begin{array}{l}\text { Since the controller and the devices are } \\
\text { connected by the standard } \\
\text { communication specifications, it is not } \\
\text { necessary to change the system just by } \\
\text { replacing the device. }\end{array}$ & $\begin{array}{l}\text { Since devices and server are } \\
\text { vertically integrated, changing the } \\
\text { devices requires changing the } \\
\text { server configuration }\end{array}$ \\
\hline
\end{tabular}

\subsubsection{Connection between Service Providers and Server}

In the architecture of "connect to server via the controller", the service provider can cooperate with one server, thereby making it possible to provide services by utilizing a plurality of devices bundled by the in-home controllers.

On the other hand, in the architecture of "connect to server directly", the service provider needs to cooperate with the servers each device vendor operates. Therefore, in the case of providing services utilizing a plurality of devices in home, a service provider is required to contract to use servers operated by a plurality of device vendors. However, if it is impossible to make a contract to use some servers operated by some device vendors, it will be impossible to provide services that control and monitor a part of the devices to the users. In addition, since standard specifications to cooperate between servers at the application level such as control commands do not exist, it is necessary for service vendors to comply with several cooperative specifications that each device vendor specifies.

Therefore, when providing services targeting multiple devices in the home, it is considered that the architecture of "connect to server via the controller" is easier to construct the system. 


\subsubsection{Introduction of Controller}

The controller installed in the house keeps the function of aggregating the information of the devices in the home and coordinating with the server. In case of using the standard communication specifications common to the communication between the controller and each device, the controller can accommodate new devices even in the case of addition or exchange of devices of different vendors. Therefore, configuration of servers and controller are not needed to change. In addition, by introducing the controller, it is unnecessary for devices with small resources to directly connect to the Internet, thus the risk of being attacked from the outside is considered to be small.

In the case where a device vendor constructs a system for the purpose of controlling and monitoring only its own device, it may be easy to construct a system based on the architecture of "connect to server directly".

\subsubsection{Introduction of IoT Device}

In the architecture of "connect to server via the controller", since the controller that aggregates each device connects to a server on the Internet, even for devices that are difficult to mount the function of connecting to the Internet, it is possible to convert to IoT device by connecting to the controller in the home network. In addition, service providers can also provide services to users who are subject to control and monitor the devices that are difficult to connect to the Internet directly.

Since it becomes unnecessary for devices to install a communication function and a security function (Joseph Bugeja et al, 2016) for connecting to the Internet, it is also possible to reduce the cost for making the device IoT.

\subsection{Realization of Various Kinds of Services}

Even if the system can be constructed in the home network, unless the user can use various services, the home network will not be used. In realizing a wide variety of services, the comparison points of the two architectures are shown in Figure 3, and the outline of the comparison is shown in Table 2. The points to be compared are "ease of implementation of a service provider", "communication state on the Internet", "real-time property of device cooperation" and "device to be controlled and monitored".

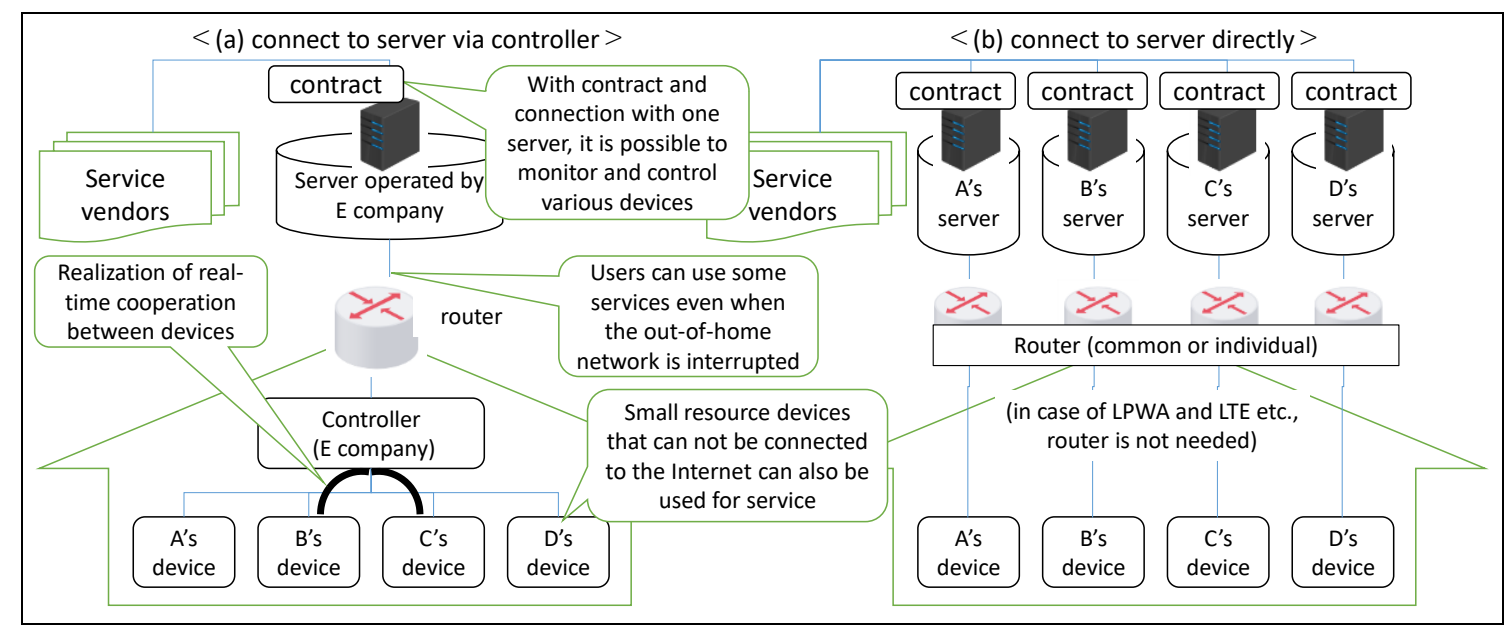

Figure 3. Comparison of architecture related to "Realization of various kinds of services" 
Table 2. Outline of Comparison of architecture related to "Realization of various kinds of services"

\begin{tabular}{|c|c|c|}
\hline & (a) Connect to server via controller & (b) Connect to server directly \\
\hline $\begin{array}{l}\text { ease of implementation of } \\
\text { service provider }\end{array}$ & $\begin{array}{l}\text { Each service provider can provide } \\
\text { services to each house by connecting } \\
\text { with one server }\end{array}$ & $\begin{array}{l}\text { "Development to connect to } \\
\text { multiple servers" and "contract } \\
\text { with server operator" are required }\end{array}$ \\
\hline $\begin{array}{l}\text { communication state on th } \\
\text { Internet }\end{array}$ & $\begin{array}{l}\text { Even when the Internet is not in service, } \\
\text { users can use some service functions } \\
\text { held by the controller }\end{array}$ & $\begin{array}{l}\text { At the time of Internet } \\
\text { disconnection, the service using } \\
\text { communication cannot be used by } \\
\text { the user }\end{array}$ \\
\hline $\begin{array}{l}\text { real-time property of devid } \\
\text { cooperation }\end{array}$ & $\begin{array}{l}\text { Since the controller controls each device } \\
\text { in the home network, cooperation } \\
\text { between devices with low delay is } \\
\text { possible }\end{array}$ & $\begin{array}{l}\text { Cooperation between servers on the } \\
\text { Internet is required, so equipment } \\
\text { control at home is delayed }\end{array}$ \\
\hline $\begin{array}{l}\text { Devices subject to control } \\
\text { and monitoring }\end{array}$ & $\begin{array}{l}\text { Service providers can also use small } \\
\text { resource devices that cannot connect to } \\
\text { the Internet for services }\end{array}$ & $\begin{array}{l}\text { Service providers can use only } \\
\text { devices that can connect to the } \\
\text { Internet for services }\end{array}$ \\
\hline
\end{tabular}

\subsubsection{Ease of Implementation of a Service Provider}

It is common to conclude a contract to connect between servers on the Internet. In the architecture of "Connect to server via controller", each service provider can provide a service to each user by linking with a server connected to the controller. On the other hand, in the architecture of "Connect to server directly", service providers need to cooperate with servers to which each device connects, and when a contract to connect with some servers cannot be made, service providers have to restrict a part of services provided to users.

At the present time, as a coordination method between servers, there is a standard method such as WebAPI, but standardization at the application level such as control command has not specified in actuality. Therefore, in the architecture of "Connect to server directly", service providers may need to implement multiple communication specifications in order to link with servers which each device vendor operates.

\subsubsection{Communication State on the Internet}

In the architecture of "Connect to server directly", when an external network such as the Internet network or mobile network is disconnected, all services provided by the service provider cannot be used. On the other hand, in the architecture of "Connect to server via controller", since the controller can control and monitor each device, it is possible to continue to use some services.

\subsubsection{Real-time Property of Device Cooperation}

First of all, the examples of "equipment cooperation at home" is shown below.

e.g. 1: When the controller detects that the power generation of photovoltaics is surplus, the controller sets the operation mode of the storage battery to the charge mode.

e.g. 2: When the controller acquires the electric power used from the smart meter and detects that the electric power is over the set value, the controller changes the state of the air conditioner.

In the architecture of "Connect to server via controller", it is the controller in the home that can monitor and control the devices. Since the controller can control the devices by using the home network after monitoring the state of the device and the measured value, it is possible to provide devices cooperation service with high real-time property. On the other hand, in the architecture of "Connect to server directly", since it is necessary to share the state of the device and control commands between servers, some delays occur in device control.

\subsubsection{Devices Subject to Control and Monitoring}

In the architecture of "Connect to server via controller", since the controller aggregates each device and connects to the server on the Internet, even in a device which is difficult to implement the function of connecting to the Internet, it becomes possible to realize IoT device by connecting to the controller in the home network. Therefore, even for service providers, it is possible to provide services to users as objects of control and monitoring for devices that are difficult to connect to the Internet. And since it is unnecessary to implement 
a communication function and a security function for connecting the device to the Internet, the cost for making the IoT devices can be lowered as well.

On the other hand, in the architecture of "Connect to server directly", implementing the function to connect to the internet may increase the cost. Also, for devices that do not have resources to implement the function for connecting to the Internet, they are out of control or monitoring objects.

\subsection{Introduction / Operation Cost}

Items to compare architectures in terms of the introduction cost at the time of system construction and the subsequent operation cost are shown in Figure 4, and outline of the comparison is shown in Table 3. As items to be compared, "Operation cost of servers", "Network line expenses", "IoT devices and controller introduction costs" can be mentioned.

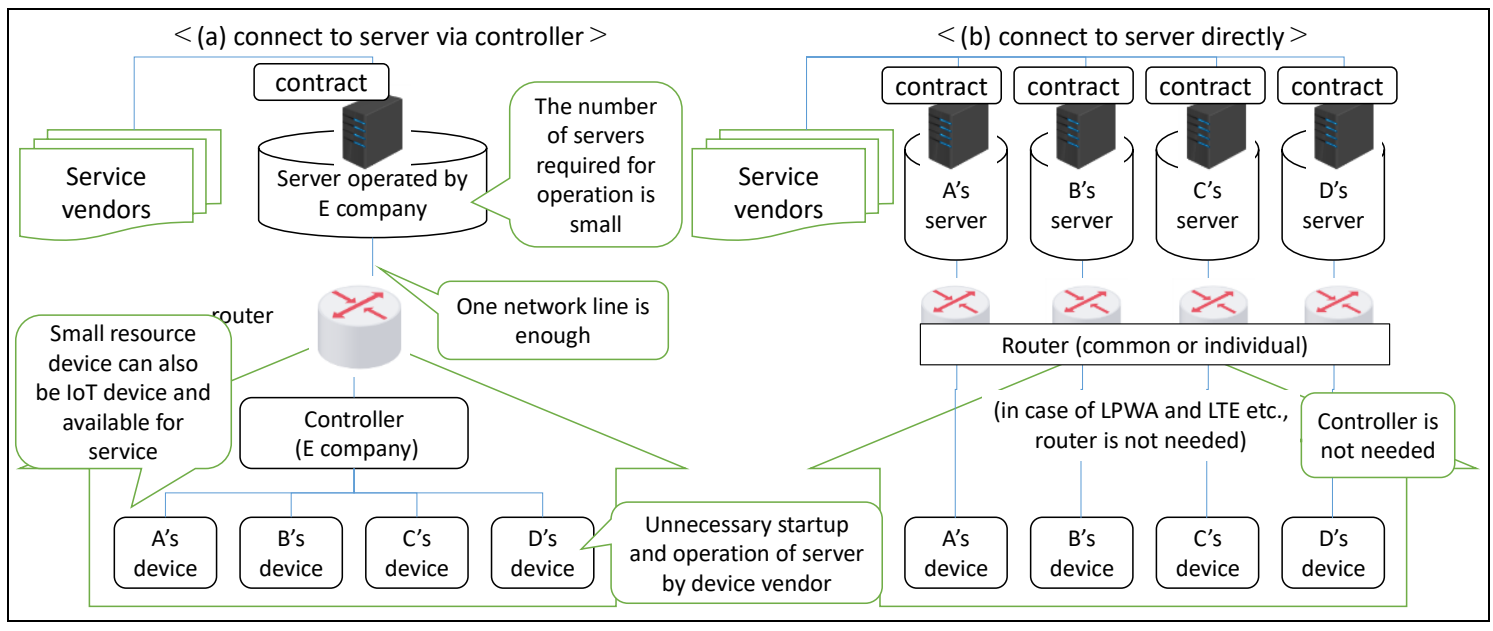

Figure 4. Comparison of architecture related to "introduction / operation cost"

Table 3. Outline of comparison of architecture related to "introduction / operation cost"

\begin{tabular}{|c|c|c|}
\hline & (a) Connect to server via controller & (b) Connect to server directly \\
\hline Operation cost of servers & $\begin{array}{l}\text { One server to which the controller } \\
\text { connects }\end{array}$ & $\begin{array}{l}\text { Number of servers connected to } \\
\text { each device at home }\end{array}$ \\
\hline Network line expenses & Basically one network line is enough. & $\begin{array}{l}\text { Depending on requirements, } \\
\text { network lines may be required for } \\
\text { each device }\end{array}$ \\
\hline $\begin{array}{l}\text { IoT devices and controllen } \\
\text { introduction cost }\end{array}$ & $\begin{array}{l}\text { Since each device does not need to } \\
\text { connect directly to the Internet, devices } \\
\text { with small resources can also become an } \\
\text { IoT device }\end{array}$ & Controller is not needed. \\
\hline
\end{tabular}

\subsubsection{Operation Cost of Servers}

In the architecture of "Connect to server via controller", a system is constructed by servers of each service provider and one server to which controller connects. On the other hand, in the architecture of "Connect to server directly", a system is constructed by servers of each service provider and a plural of servers which each device vendor operates. Assuming that the amount of data handled by each server is almost the same, the architecture of "Connect to server via controller" seems to be able to reduce the operation cost of the server because the number of servers to be operated is smaller. 


\subsubsection{Network Line Expenses}

In case of the architecture of "Connect to server via controller", since only the controller connects to the Internet, if there is only one network line, the devices which connect to the controller can be made IoT devices, and the service provider can provide the service of the home network to the user. If users already have an Internet environment at home, users can build it without additional communication cost by using that environment. On the other hand, in case of the architecture of "Connect to server directly", similarly there are cases in which a system can be constructed with one network line. However, depending on the product, there are cases where the use of exclusive lines or independent networks is must. In addition, when introducing IoT devices using communication lines such as LPWA and LTE, the cost of communication lines may be required for each device.

\subsubsection{IoT Devices and Controller Introduction Costs}

In the architecture of "Connect to server via controller", each device only needs to implement a home network function for communicating with a controller in the home, and it is possible to become an IoT device at low cost. In addition, inexpensive devices with small resources that are difficult to install communication functions and security functions for connecting to the Internet can also be used as IoT devices. In the architecture of "Connect to server directly", since no controller is required, the cost of the controller is unnecessary. However, as described above, it is necessary to install a function of Internet connection, and only devices having a certain level of resources can be handled.

\section{FEASIBILITY OF ARCHITECTURE VIA CONTROLLER}

From the comparison of the architecture shown in Chapter 2, when providing various services to control and monitor a large number of devices in the home, it is necessary to construct a home network by converting the equipment into IoT in the architecture via the controller, it turned out that it is better to construct a home network by IoT device in the architecture of "Connect to server via controller" than in the architecture of "Connect to server directly".

For services that device vendors control and monitor only their own equipment, it can be realized with the architecture of "Connect to server directly". However, restrictions are large when trying to deal with a wide variety of services over the medium to long term.

In this chapter, the "standard communication specifications between controller and device in the home" which is important to realize the architecture of "Connect to server via controller" and the devices which is compatible with the standard communication specification are introduced.

\subsection{ECHONET Lite which is International Standard Specifications}

There are various international standard communication specifications such as ECHONET Lite (ISO/IEC 14543-4-3 / IEC 62394), KNX (ISO/IEC 14543-3) and ZigBee etc. in the communication specifications between the controller and the IoT devices. In this paper, ECHONET Lite is described as an example of standard communication specifications. The communication specification of ECHONET Lite is ISO / IEC 14543-4-3, and the control commands of devices is IEC 62394, both of which are international specifications.

Main features of ECHONET Lite are "Transport-free communication specifications that can use existing standard transmission media such as Ethernet, Wi-Fi, etc." "Specify detailed control commands of more than 110 types of devices targeting general households, small and medium-sized buildings, and small and medium-sized stores", "High interoperability by specifying how to use concrete ECHONET Lite at the application level" (Takashi Murakami et al., 2016) and "A wide variety of services can be realized by architecture via controller". Numerous IoT devices and controllers compatible with ECHONET Lite are already on the market. 


\subsection{IoT Devices and Controller which complied with ECHONET Lite}

Number of certification acquired is shown in Figure 5 (referred to https://echonet.jp/number-of-certificationacquired/). Since the launch of certification system of ECHONET Lite in 2012, more than 600 applications have been certified by the end of September 2018. In addition, the shipment quantity of ECHONET Lite devices is cumulative total of more than 17 million units. In Japanese smart energy meter, which is the key device to energy management, ECHONET Lite is installed in approximately 28 million smart energy meters as of March 2017 (finally 77 million smart energy meters).

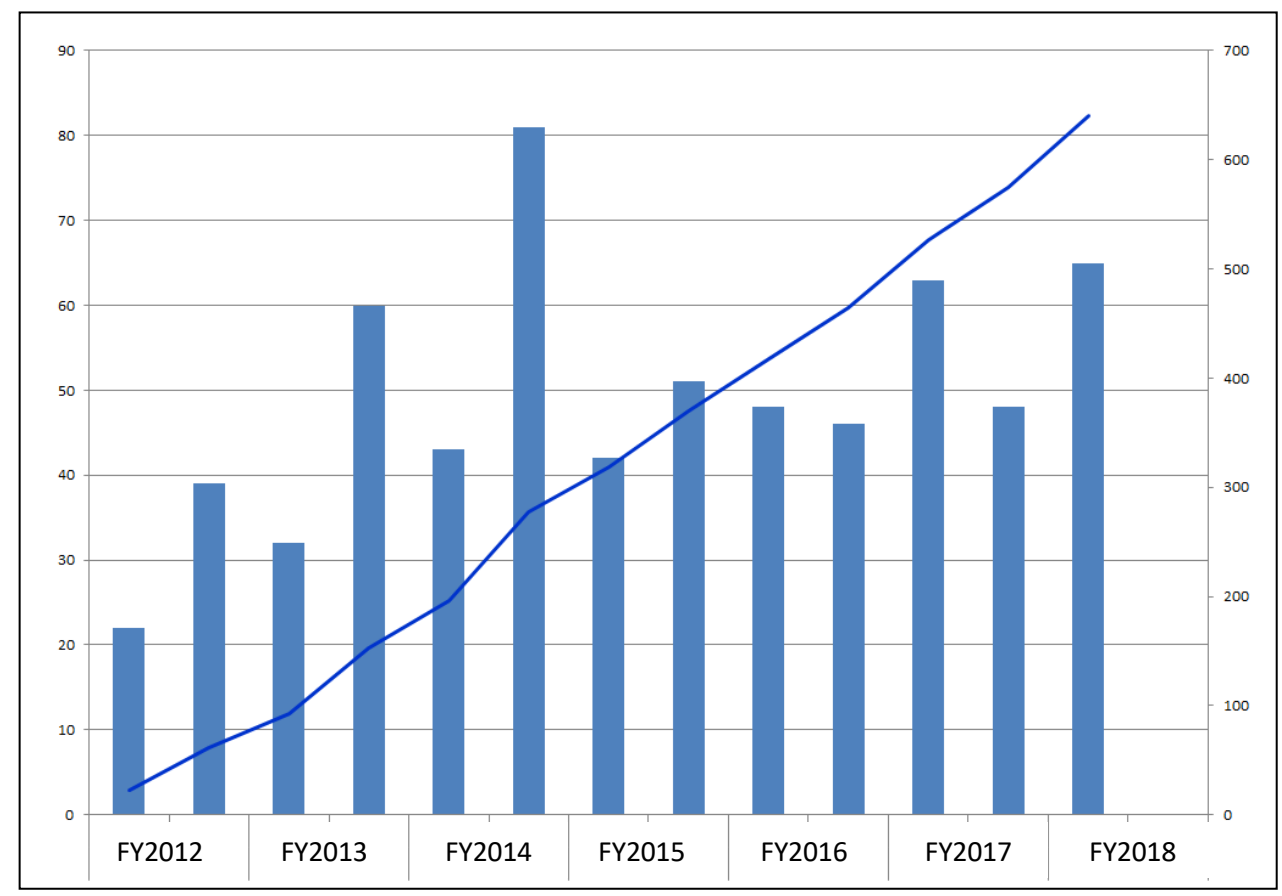

Figure 5. Number of certification acquired and shipment quantity

In addition, the controller certified by ECHONET Lite and shipped to the market are shown in Figure 6 and the devices certified by ECHONET Lite and shipped to the market are shown in Figure 7.

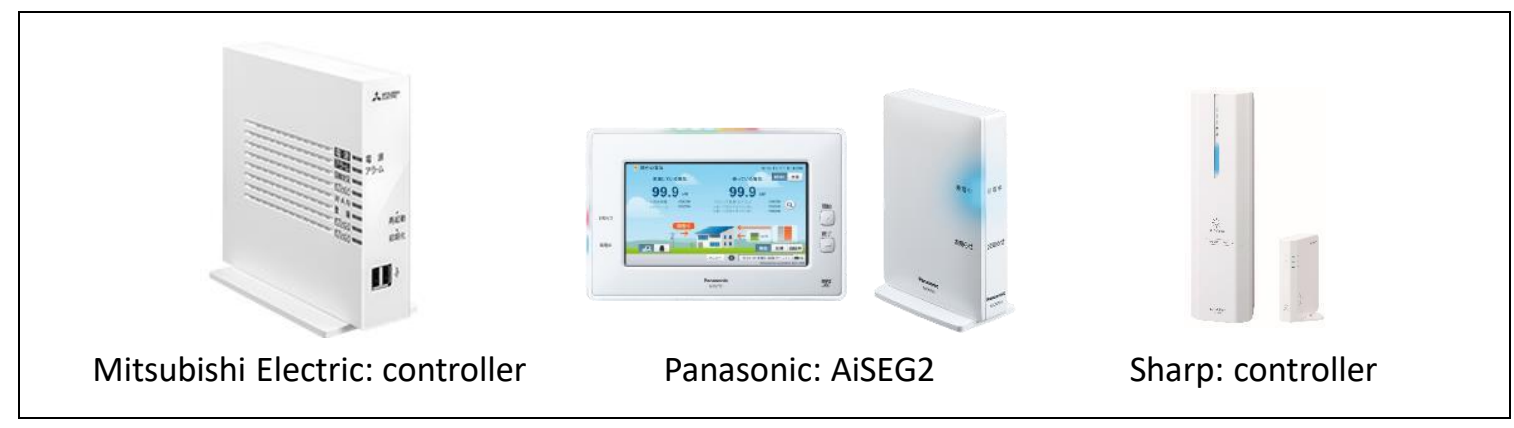

Figure 6. Examples of ECHONET Lite certified controllers 


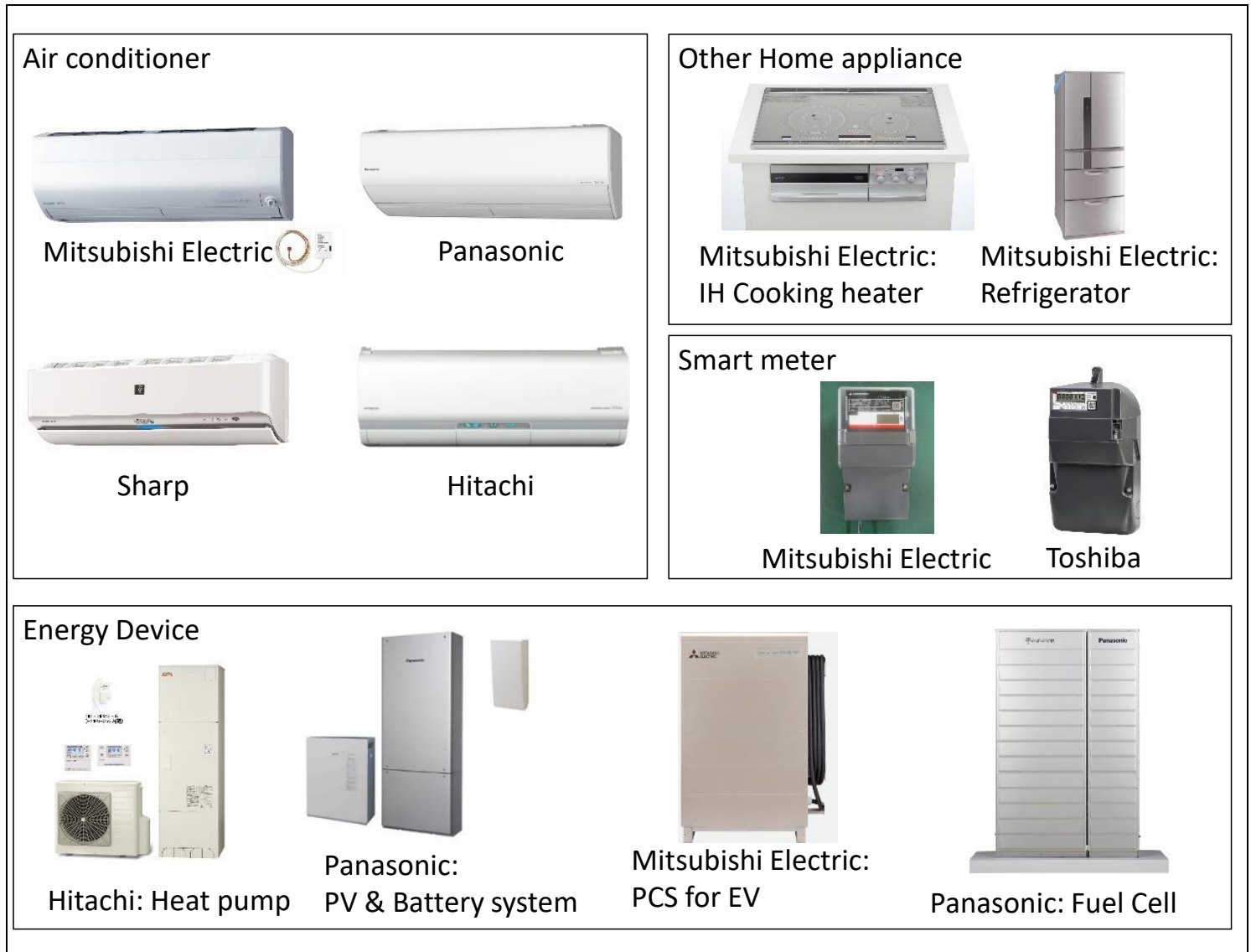

Figure 7. Examples of ECHONET Lite certified devices

\section{CONCLUSION}

The architecture of "Connect to server via controller" and the architecture of "Connect to server directly" have been compared from the viewpoint of the IoT devices in the home. As a result, it was confirmed that the architecture of "Connect to server via controller" can enjoy various merits in order to provide various services to users. In the architecture of "Connect to server directly", it is possible to easily construct a system to perform services targeting only monitoring and control of specific device. However, it is difficult to extend the system when providing services targeting the entire house to the user. Furthermore, in order to realize the architecture of "Connect to server via controller", commercialization of controllers and IoT device compliant with standard specifications becomes important. In fact, with the "Connect to server via controller" architecture, there are marketed services linked with Google Home, Amazon Alexa, etc. (Cu et al., 2018) And one example of IoT devices is devices and controllers implemented with the international standard ECHONET Lite (ISO / IEC 14543-4-3, IEC 62394).

\section{ACKNOWLEDGEMENT}

The authors would like to thank the ECHONET Consortium members for cooperating in the development of standard specifications, promoting standardization, and providing various data any time. 


\section{REFERENCES}

Biljana L. and Kire V., 2017. A review of Internet of Things for smart home: Challenges and solutions. Cleaner Production, Vol. 140, Part 3, pp.1454-1464.

Charlie, W. et al, 2014. Smart homes and their users: a systematic analysis and key challenges. Springer-Verlag, London, UK.

$\mathrm{Cu}$ Pham, Yuto Lim, and Yao Tan, 2018. A Platform for Integrating Alexa Voice Service into ECHONET-Based Smart Homes, 2018 IEEE International Conference on Consumer Electronics-Taiwan, Taichung, Taiwan, pp.1-5

ECHONET, https://echonet.jp/english/, (Accessed January, 2019).

Joseph Bugeja et al, 2016. On privacy and Security Challenges in Smart Connected Homes. Proceedings of European Intelligence and Security Informatics Conference 2016, Uppsala, Sweden, pp.172-175.

KNX, https://www.knx.org/knx-en/for-professionals/index.php, (Accessed January, 2019).

Takashi Murakami, Hiroshi Sugimura and Masao Isshiki, 2016. Application of ECHONET Lite which is open standard into energy management system. 2016 IEEE International Conference on Consumer Electronics, Las Vegas, NV, USA, pp.493-496.

ZigBee, http://www.zigbee.org/, (Accessed January, 2019). 